CARDIOVASCULAR MEDICINE

\title{
Long term complications in single and dual chamber pacing are influenced by surgical experience and patient morbidity
}

\author{
F Eberhardt, F Bode, H Bonnemeier, F Boguschewski, M Schlei, W Peters, U K H Wiegand
}

Heart 2005;91:500-506. doi: 10.1136/hrt.2003.025411

See end of article for authors' affiliations

Correspondence to:

Dr F Eberhardt,

Universitätsklinik

Schleswig Holstein,

Campus Luebeck,

Medizinische Klinik II,

Ratzeburger Allee 160,

23538 Luebeck, Germany;

eberhard@uni-luebeck.de

Accepted 7 June 2004

\begin{abstract}
Objective: To determine how short and long term complication rates after pacemaker implantation are influenced by patient morbidity, operator experience, and choice of pacing system.

Design: Retrospective analysis of 1884 patients who received VVI $(n=610)$, VDD $(n=371)$, or DDD devices ( $n=903$ ) between 1990 and 2001. Follow up period was 64 (34) months. The influence of age, sex, coronary artery disease, myocardial infarction, reduced left ventricular (LV) function, right ventricular (RV) dilatation, atrial fibrillation, device type, and operator experience on operation time and complication rate were analysed.

Results: Operation time was prolonged in patients with coronary artery disease, inferior myocardial infarction, reduced LV function, and RV dilatation. Implantation of DDD pacemakers prolonged operation time, particularly among operators with a low or medium level of experience. The overall complication rate was $4.5 \%$. Sixty seven per cent of these complications occurred within the first three months. Complication rate was increased by age, reduced LV function, and RV dilatation. Implantation of DDD systems led to a higher complication rate $(6.3 \%)$ than implantation of VVI $(2.6 \%)$ or VDD pacemakers $(3.2 \%)$. These differences were present only among operators with a low or medium level of experience.

Conclusions: Operation time and complication rate increased with age, impaired LV function, and RV dilatation. Complication rates were higher with DDD than with VVI or VDD implantation and were excessive among inexperienced but not experienced operators.
\end{abstract}

A $\mathrm{V}$, atrioventricular; CTOPP, Canadian trial of physiologic pacing; LV, left ventricular; MOST, mode selection trial in sinus node dysfunction; PASE, pacemaker selection in the elderly; RV, right ventricular

Investigators in the CTOPP (Canadian trial of physiologic pacing) study reported an overall incidence of perioperative complications of $9.0 \%$ for dual chamber pacing compared with $3.8 \%$ for ventricular pacing among patients with atrioventricular (AV) block. ${ }^{1}$ Although implantation of dual chamber devices is technically more challenging and time consuming, the difference in complication rates between single and dual chamber pacing is not consistent in all studies. ${ }^{1-11}$ In another large randomised study, MOST (mode selection trial in sinus node dysfunction), the 30 day complication rate of $4.8 \%$ was substantially lower for dual chamber pacemaker implantation. ${ }^{4}$ The Danish pacemaker register, the largest available database so far, reported an average overall perioperative complication rate of 3.5\% over five years. ${ }^{5}$ Sixty five per cent of the pacemakers implanted were DDD pacemakers. Although some of these differences in complication rates may be explained by the closer follow up of patients in prospective randomised trials, different levels of operator experience may also have an important role..$^{8-10}$ Some authors argue that in the hands of experienced operators dual chamber pacing carries no higher risk of early complications than single chamber pacing. ${ }^{10}$ Another factor that may have a substantial impact on complication rates is patient co-morbidity, particularly since a significant number of patients in large trials are affected by coronary heart disease, reduced left ventricular (LV) function, or atrial fibrillation. ${ }^{1-4}$ For patients with AV block, VDD pacing may be an interesting alternative to achieve physiological pacing at a lower complication rate than DDD pacing. ${ }^{12-14}$ However, long term complication rates of VDD pacing have not been systematically investigated so far. We analysed 1884 consecutive patients from our institutional database to determine how short and long term complication rates were influenced by patient morbidity, operator experience, and choice of pacing system.

\section{PATIENTS AND METHODS}

\section{Patient characteristics and pacing systems}

We retrospectively evaluated 1884 patients who received either a VVI $(n=610)$, a VDD $(n=371)$, or a DDD $(\mathrm{n}=903)$ pacemaker system at the University Hospital of Luebeck, Germany between 1990 and 2001. All patients underwent 12 lead ECG, chest radiography, echocardiography, and a pacemaker follow up before discharge from hospital. Table 1 lists patient characteristics and pacing systems used, as well as echocardiographic criteria for reduced LV function and right ventricular (RV) dilatation. Coronary artery disease was defined as being present if angiographic criteria were fulfilled or the patient had a history of myocardial infarction. In the VVI group, patients were significantly older than patients with DDD or VDD pacemakers and had a higher incidence of structural heart disease and atrial fibrillation.

\section{Implantation procedure}

Operations were performed in an operating theatre according to our institutional standard, which remained unchanged during the study period. Briefly, a pectoral incision was made under local anaesthesia. Venous access was achieved by cephalic cut down whenever possible. If preparation of the cephalic vein failed or insertion of the second lead was impossible, the subclavian vein was punctured directly. An atrial lead position was accepted when the potential amplitude was $>2.0 \mathrm{mV}$ and the pacing threshold was $<1.5 \mathrm{~V}$ at a $0.5 \mathrm{~ms}$ pulse duration. Smaller values were accepted only when the required criteria had not been met at four different anatomical positions. A ventricular lead position was accepted when the potential amplitude was 


\begin{tabular}{|c|c|c|c|c|}
\hline & $\begin{array}{l}\text { DDD } \\
(n=903)\end{array}$ & $\begin{array}{l}\text { VDD } \\
(n=371)\end{array}$ & $\begin{array}{l}\text { VVI } \\
(n=610)\end{array}$ & $\begin{array}{l}\text { Total } \\
(n=1884)\end{array}$ \\
\hline \multicolumn{5}{|l|}{ Patient characteristics } \\
\hline Age (years) & $70.3(12.0)^{*} \dagger$ & $67.7(14.3) \ddagger$ & $77.4(9.1)$ & $72.1(12.3)$ \\
\hline Male sex & $53.7 \%{ }^{*}+$ & $60.1 \% \ddagger$ & $51.8 \%$ & $54.4 \%$ \\
\hline Coronary artery disease & $52.4 \% \dagger$ & $53.1 \% \ddagger$ & $64.8 \%$ & $56.6 \%$ \\
\hline Inferior myocardial infarction & $13.0 \% \dagger$ & $13.2 \% \ddagger$ & $21.3 \%$ & $15.7 \%$ \\
\hline Reduced LV function§ & $25.5 \% \dagger$ & $23.7 \% \ddagger$ & $32.8 \%$ & $27.5 \%$ \\
\hline Right ventricular dilatation & $13.5 \% \dagger$ & $16.7 \% \ddagger$ & $24.1 \%$ & $17.6 \%$ \\
\hline \multicolumn{5}{|l|}{ Indication for pacing } \\
\hline Sinus node disease & $55.5 \%{ }^{*} \dagger$ & & $26.4 \%$ & $35.1 \%$ \\
\hline $\mathrm{AV}$ conduction disturbance & $57.9 \% * \dagger$ & $100 \% \ddagger$ & $81.1 \%$ & $73.7 \%$ \\
\hline Paroxysmal/persistent AF & $27.2 \%^{*} \dagger$ & $0.8 \% \ddagger$ & $18.5 \%$ & $19.2 \%$ \\
\hline Permanent $\mathrm{AF}$ & $0 *+$ & $0 \ddagger$ & $46.9 \%$ & $15.2 \%$ \\
\hline \multicolumn{5}{|l|}{ Level of operator experience } \\
\hline Number of implantations & $176(143)^{*} \dagger$ & $156(125)$ & 149 (109) & $163(131)$ \\
\hline Level 1 & $12.2 \%$ & $16.4 \%$ & $15.3 \%$ & $14.1 \%$ \\
\hline Level 2 & $33.0 \% \dagger$ & $28.3 \%$ & $26.7 \%$ & $30.0 \%$ \\
\hline Level 3 & $54.8 \%$ & $55.3 \%$ & $58.0 \%$ & $55.9 \%$ \\
\hline \multicolumn{5}{|l|}{ Pacemaker systems } \\
\hline Bipolar atrial leads & $96.5 \%$ & NA & NA & NA \\
\hline Bipolar ventricular leads & $95.5 \%{ }^{*} \dagger$ & $87.1 \%$ & $82.8 \%$ & $89.7 \%$ \\
\hline Active fixation lead (atrium)** & $100 \%$ & NA & NA & NA \\
\hline Active fixation lead (ventricle) & $10.3 \%{ }^{*} \dagger$ & $0 \ddagger$ & $21.1 \%$ & $11.8 \%$ \\
\hline Subclavian vein puncture & $85 \%{ }^{*} \dagger$ & $31.8 \%$ & $35.4 \%$ & $58.4 \%$ \\
\hline Rate responsive systems & $47.4 \%{ }^{*} \dagger$ & $86.8 \% \ddagger$ & $26.4 \%$ & $48.3 \%$ \\
\hline Follow up (months) & $64.3(32.0)^{*}$ & $66.7(27.3) \ddagger$ & $59.8(36.9)$ & $64.0(34.1)$ \\
\hline \multicolumn{5}{|c|}{ 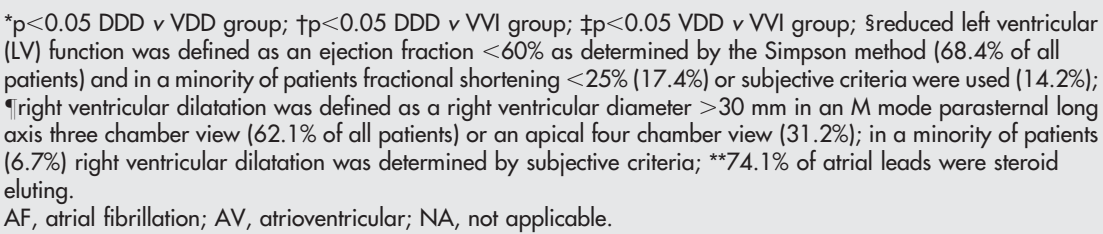 } \\
\hline
\end{tabular}

$>6 \mathrm{mV}$ and the pacing threshold was $<1 \mathrm{~V}$ at $0.5 \mathrm{~ms}$. The atrial dipole of VDD leads was positioned at a low, medium, and high atrial position according to anterior-posterior fluoroscopy. The position that achieved the highest atrial potential at deep inspiration was chosen. When the atrial amplitude was $<0.8 \mathrm{mV}$ at any position, an active fixation lead was implanted. ${ }^{15}$ For all pacing systems, the implantation standard was not fulfilled in 3.4\% of all implantations. Antiplatelet medication was continued perioperatively. Oral anticoagulation was discontinued 3-5 days before the operation and replaced by intravenous heparin whenever stringent anticoagulation was indicated. Heparin was discontinued two hours before the operation and restarted afterwards. Prophylactic antibiotics were given perioperatively in almost all cases; however, the antibiotic regimen was not consistent during the study period. In the majority of cases, a first generation cephalosporin was administered once preoperatively and three times postoperatively. Other antibiotics used were flucloxacillin, vancomycin, and ampicillin plus sulbactam, sometimes for as long as three days postoperatively at the operator's discretion.

Table 2 Influence of patient characteristics on operation and fluoroscopy times

\begin{tabular}{|c|c|c|}
\hline & $\begin{array}{l}\text { Prolongation of } \\
\text { operation time } \\
\text { (minutes) }\end{array}$ & $\begin{array}{l}\text { Prolongation of } \\
\text { fluoroscopy time } \\
\text { (minutes) }\end{array}$ \\
\hline Patient age $>74$ years & $1.2(-1.4$ to 03.2$)$ & $0.4(-0.2$ to 1.0$)$ \\
\hline Male sex & $0.3(-1.5$ to 02.0$)$ & $-0.2(-0.6$ to 0.2$)$ \\
\hline Coronary artery disease & $2.6(0.8$ to 04.3$)$ & $0.5(0.0$ to 0.9$)$ \\
\hline Inferior myocardial infarction & 8.2 (5.8 to 10.7$)$ & $1.6(1.0$ to 2.2$)$ \\
\hline Reduced LV function & $11.0(9.1$ to 12.9$)$ & 2.1 (1.7 to 2.6$)$ \\
\hline Right ventricular dilatation & 16.2 (14.1 to 18.3 ) & $3.0(2.5$ to 3.5$)$ \\
\hline
\end{tabular}

\section{Operator experience}

Before inclusion into the study, operators had to have performed at least 25 implantations under direct supervision of an experienced operator. Operator experience was assessed by the number of implantation that he or she had performed before the study implantation. For clarity, three levels of experience were defined, which are supported by previous studies that investigated the influence of operator experience on complication rates $^{8-10}$ : fewer than 50 implantations denoted a low (level 1), between 50 and 100 implantations a medium (level 2), and more than 100 implantations a high level of experience (level 3). Operators who implanted DDD pacemakers were significantly more experienced than operators who implanted VDD or VVI pacemakers (table 1).

\section{Complications}

A complication was defined as an event that required either surgical revision-that is, lead dislodgment, loss of sensing or exit block, cardiac perforation, pocket haematoma, infection, or erosion-or an invasive procedure, such as a pleural catheter for pneumothorax or drainage of a haemothorax. Early complications were those that occurred within the first three months after implantation and late complications occurred thereafter.

\section{Follow up}

Follow up was performed at our outpatient clinic at six month intervals. Mean (SD) follow up period was 64 (34) months.

\section{Statistical analysis}

SPSS for Windows version 10.0.1 (SPSS Inc, Chicago, Illinois, USA) was used for statistical analysis. Data are expressed as mean (SD). For normally distributed variables, univariate data were compared between the three groups by a one way 

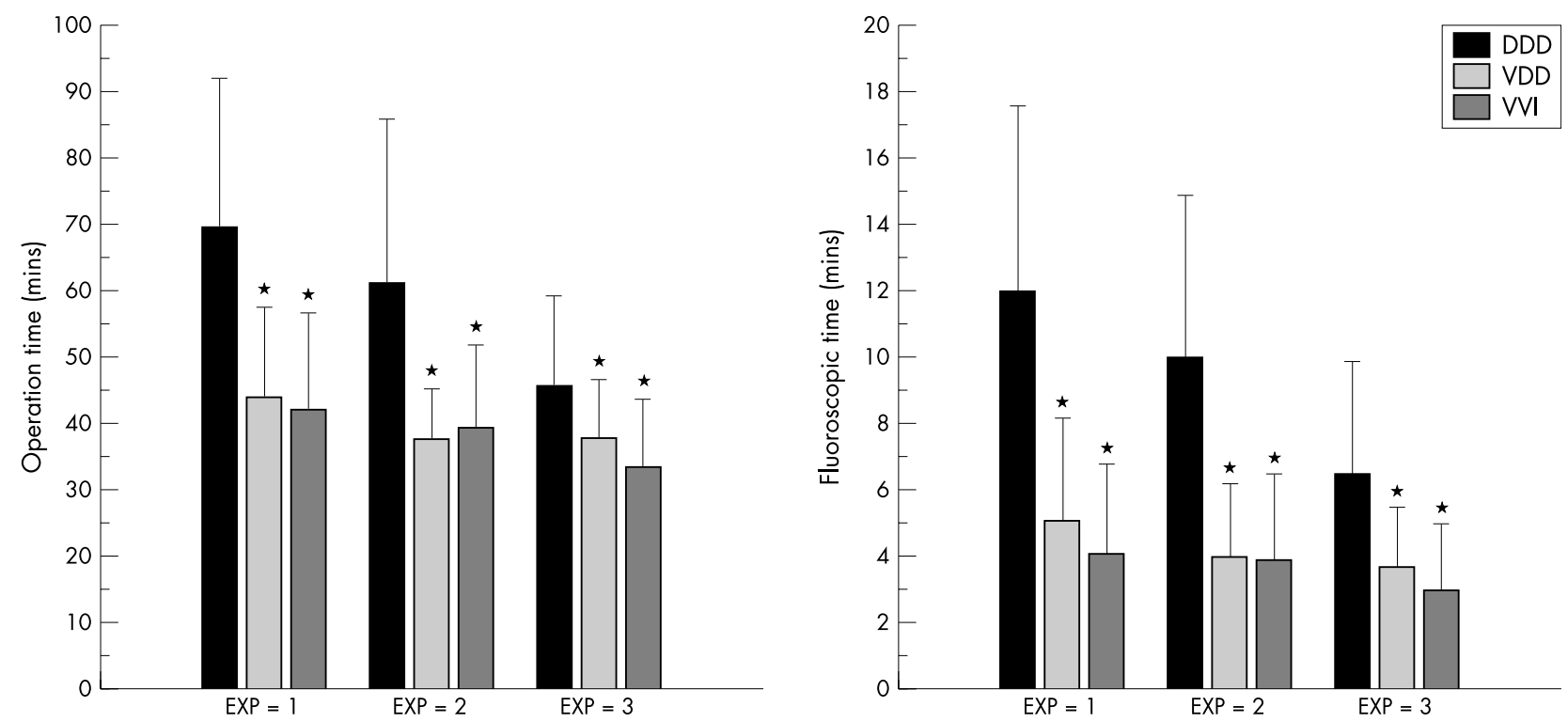

Figure 1 Unadjusted comparison of operation and fluoroscopy times related to device and surgical experience of the operator (level 1, inexperienced; level 2, medium level of experience; level 3, high level of experience). Differences between DDD and VDD or VVI pacemaker implantation decreased with an increasing level of surgical experience but remained significant. ${ }^{*} p<0.05$.

analysis of variance with a post hoc analysis by Scheffe's method. Non-normally distributed variables were compared with the Kruskal-Wallis test with a post hoc Neminye procedure. Frequencies were compared by $\chi^{2}$ test or in subgroups with low sample size by Fisher's exact test. Differences in operation and fluoroscopy times of the pacemaker systems were evaluated in a multivariate linear regression model including age, sex, coronary artery disease, history of inferior myocardial infarction, reduced LV function, RV dilatation, and atrial fibrillation. A Cox regression hazard model was used to compare the cumulative complication hazard between the pacemaker system. Data were analysed both unadjusted and adjusted for the clinical covariates mentioned above. A probability value of $\mathrm{p}<0.05$ was considered significant.

\section{RESULTS}

\section{Implantation}

Operation and fluoroscopy time were significantly prolonged in patients with coronary artery disease, inferior myocardial infarction, reduced LV function, and RV dilatation (table 2). Implantation and fluoroscopy times were significantly influenced by the implanted pacemaker device and by the experience of the operator (fig 1). Implantation of a DDD pacemaker prolonged operation and fluoroscopy times particularly among operators with a low (level 1) or a medium level (level 2) of experience, whereas the device dependent difference in implantation times was substantially lower among highly experienced operators (fig 1). Implantation times did not differ significantly between VVI and VDD pacemaker implantation independent of the level of
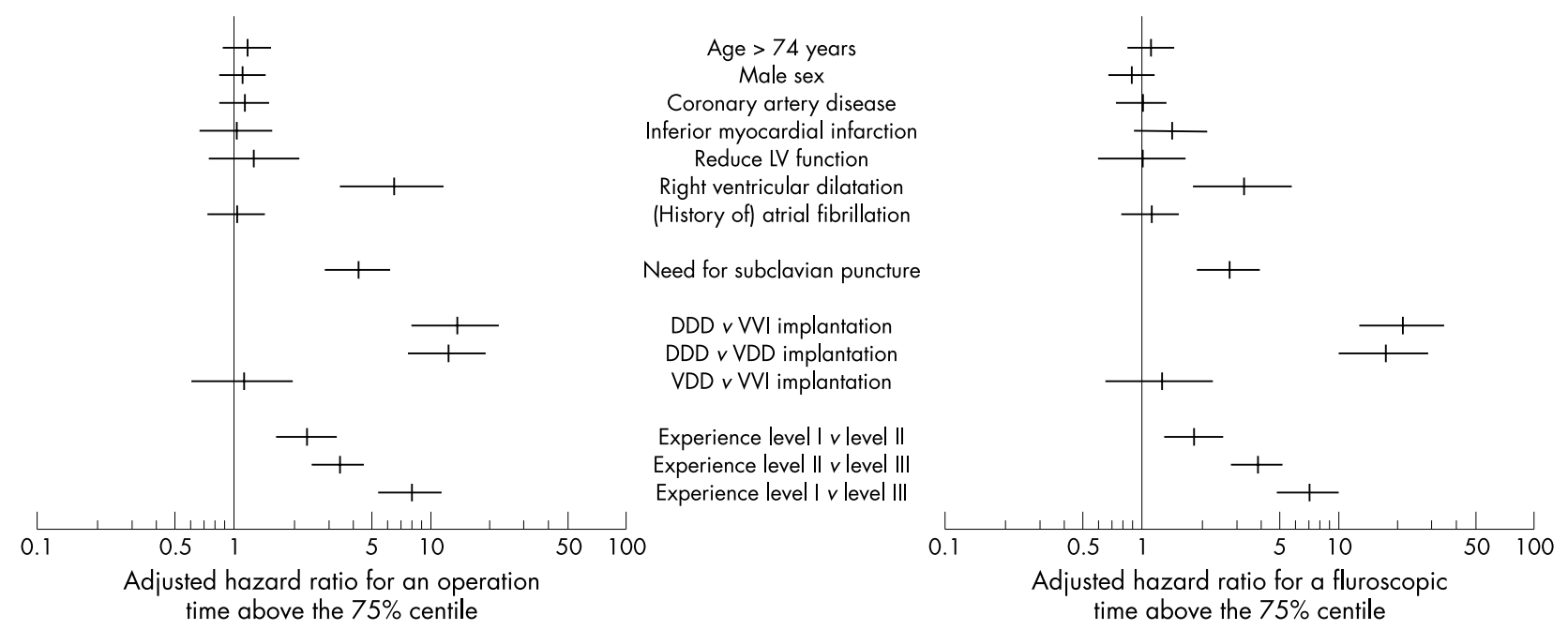

Figure 2 Independent predictors for a prolonged (left) operation or (right) fluoroscopy time identified by multivariate logistic regression analysis. Right ventricular infarction, need for puncture of the subclavian vein, DDD pacemaker implantation, and the level of surgical experience were independently predictive for both operation and fluoroscopy times that were prolonged above the $75 \%$ centile of the patient group. LV, left ventricular. 


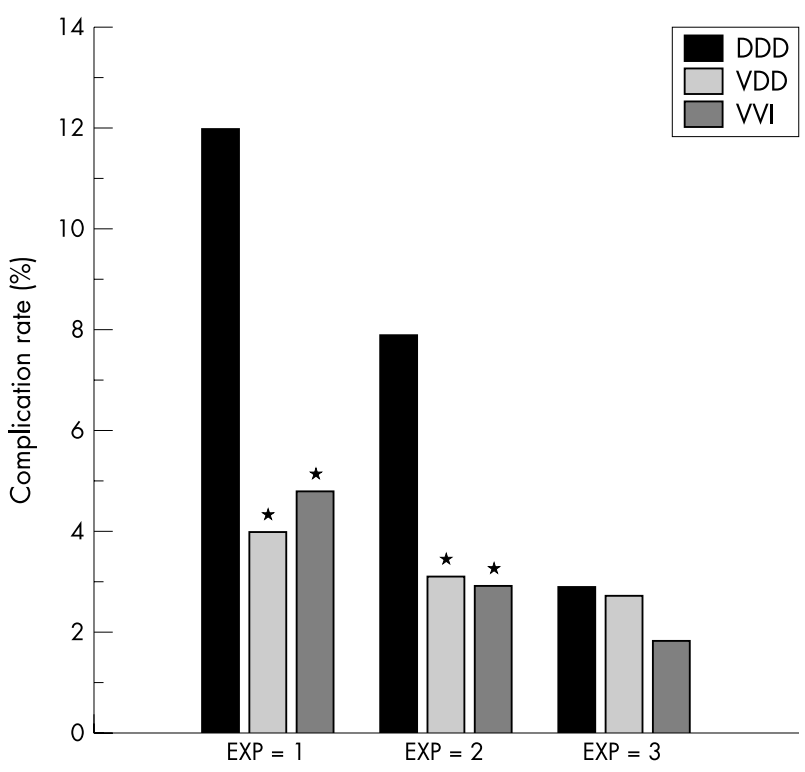

Figure 3 Univariate comparison of complication rate related to device and surgical experience. There were more complications of implanted DDD pacemakers than of VDD and VVI pacemakers implanted by surgeons with an experience level of 1 or $2(p<0.05)$ but not by experienced operators (level 3). Complication rates of VDD and VVI pacemakers did not differ significantly for any experience level. *Significantly lower than with DDD $(p<0.05)$.

operator experience. Implantation time decreased with the operators' cumulative number of implantations; however, this decrease was more pronounced for DDD pacemaker implantation.

The need for subclavian vein puncture increased mean implantation time by 13.5 (95\% confidence interval (CI) 12.1 to 14.9) minutes and fluoroscopy time by 2.6 (95\% CI 2.3 to 2.9) minutes, regardless of which pacemaker system was implanted. The frequency of subclavian, as opposed to cephalic, access was not significantly influenced by the level of experience but was higher in DDD pacemaker implantation (table 1).

In a multivariate regression analysis, implantation of a DDD pacemaker, level of experience, need for subclavian vein puncture, and presence of RV dilatation were identified as independent predictors for prolonged implantation and fluoroscopy times (fig 2).

\section{Complication rates \\ Early complications}

The overall rate of complications with need for surgical revision was $4.5 \%$. Sixty seven per cent of these complications occurred within three months after implantation. Reasons for an early surgical reintervention were lead dislodgment, loss of sensing or exit block of the atrial lead $(2.0 \%$ of DDD pacemaker implantations), pocket bleeding $(0.6 \%)$, pneumothorax $(0.6 \%)$, microdislocation of the ventricular lead $(0.6 \%)$, and myocardial perforation by the ventricular lead $(0.1 \%)$. Associated with the higher incidence of subclavian vein puncture, the rate of pneumothoraces was significantly higher with DDD pacemaker implantation (1.1\%) than with VDD and VVI implantation $(0.1 \%, \mathrm{p}<0.001)$. If the implantation standards for pace and sense characteristics were not fulfilled, the overall complication rate was $6.3 \%$ compared with $4.5 \%(\mathrm{p}=0.53)$.

\section{Late complications}

During further follow up, the annual complication rate was $0.5 \% /$ year. Reasons for later surgical reintervention were

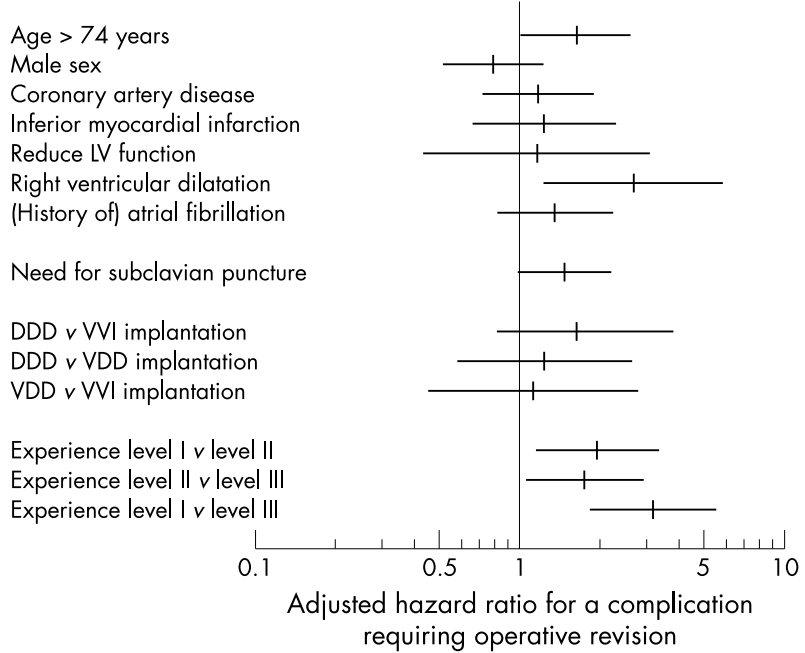

Figure 4 Independent predictors for an increased complication hazard identified by multivariate Cox regression analysis. Patient age $>74$ years, right ventricular dilatation, and the level of surgical experience were independently predictive for an increased complication hazard. *Significantly lower than with DDD $(p<0.05)$.

sensing failure or exit block of the ventricular $(0.7 \%)$ or the atrial lead $(0.2 \%$ of DDD implantations), symptomatic atrial undersensing of VDD devices (0.5\% of VDD implantations), sinus node syndrome in patients with VDD devices $(0.3 \%$ of VDD implantation), pacemaker syndrome in VVI pacing ( $0.6 \%$ of VVI implantations), defects of lead insulation $(0.4 \%)$, pocket or lead infection $(0.3 \%)$, erosion of the pacemaker pocket $(0.2 \%)$, and late perforation of the ventricular lead $(0.05 \%)$.

\section{Influence of patient morbidity and operator experience}

The incidence of complications was increased among patients aged $>74$ years $(5.7 \% \vee 3.1 \%, p=0.006)$ or with reduced LV function $(7.9 \% v 3.2 \%, \mathrm{p}<0.001)$ or RV dilatation $(10.4 \%$ $v 3.1 \%, \mathrm{p}<0.001$ ). (Micro)dislocation, sensing failure, and exit block of the ventricular lead occurred more often among patients with RV dilatation: $3.3 \%$ compared with $0.5 \%$ among patients with a normal RV diameter $(\mathrm{p}<0.001)$. Pneumothoraces or lead fractures were not observed when the cephalic route was used; however, after subclavian vein puncture, pneumothoraces occurred in $1.0 \%$ and insulation defects in $0.7 \%$ of patients. Implanters with a low level (level 1) of experience had a substantially higher complication rate $(9.1 \%)$ than operators with medium (level 2: 5.8\%) or high level of experience (level 3: 2.7\%, p $<0.05$ for all comparisons). After more than 200 implantations, the complication rate decreased to $1-2 \%$; a further increase in number of implantations did not substantially lower the complication rate.

In DDD pacemaker implantation, the complication rate was twice as high as in VVI or VDD pacemaker implantation: $6.3 \%$ versus $2.6 \%(p<0.001)$ and $3.2 \%(p=0.03)$, respectively. Device dependent differences were significant only when the operation was performed by implanters with a low or medium experience level rather than by highly experienced implanters (fig 3).

In a multivariate Cox regression analysis, patient age $>74$ years, the presence of RV dilatation, and the experience of the implanter were independently predictive for the occurrence of a complication (fig 4). The adjusted hazard ratio for the cumulative experience-the number of pacemakers implanted regardless of the type of pacing system-was 
0.994 (95\% CI 0.990 to 0.997 ). This accounts for a relative risk reduction of $0.6 \%$ for each implantation.

For implanters with low and medium experience levels, the adjusted complication hazard of a DDD pacemaker implantation was significantly higher than for VDD or VVI implantation (fig 4). However, this difference was not observed for highly experienced implanters. After correction for surgical experience, device dependent differences were no longer significant.

\section{DISCUSSION}

Patient age and structural diseases of the LV and particularly the RV influence operation times and complication rates of pacemaker implantation. Although in general complications rates are lower with higher levels of experience, ${ }^{8-10}$ a device specific analysis in our study showed that DDD pacemaker implantation by operators with a low level of experience carries a considerably higher risk of complications than VDD or VVI pacemaker implantation. Among experienced implanters we detected no device specific differences in complication rates. The learning curve for VDD pacing seems to be similar to that for VVI pacing.

\section{Operation and fluoroscopy times}

In a univariate analysis the presence of coronary artery disease, history of inferior myocardial infarction, reduced LV function, and RV dilatation were predictive for prolonged operation and fluoroscopy times. These factors are interrelated-for example, RV dilatation may be due to RV involvement after inferior myocardial infarction or to pulmonary artery hypertension after impaired systolic or diastolic LV function. ${ }^{16}$ Owing to this interrelation, RV dilatation remained the only structural disease that significantly increased operation time in a multivariate analysis. Several reasons may contribute to this finding: stable ventricular lead fixation may be more difficult in a dilated and thin RV; and unfavourable pacing thresholds and signal amplitudes may cause more attempted lead placements particularly after $\mathrm{RV}$ infarction. ${ }^{17} \mathrm{RV}$ dilatation is often accompanied by tricuspid valve regurgitation and right atrial dilatation, which may additionally prolong placement times of atrial and ventricular leads. Interestingly, sinus node disease was not associated with long operation times. It has been argued that sinus node disease is accompanied by structural right atrial disease, which would presumably make the placement of the atrial lead more difficult, as the signal quality deteriorates in the presence of right atrial disease. ${ }^{18}$ On the contrary, we found that operation times tended to be longer with AV nodal disease, most probably due to the association with structural heart ventricular disease. The need for puncture of a subclavian vein also prolonged operation and fluoroscopy times. This is attributable to the institutional standard that preparation of the cephalic vein be preferred as the access route for lead placement. Previous reports have shown that using the subclavian access as the route of choice shortens implantation times at the cost of a higher complication rate. $^{78}$ The rate of pneumothoraces after puncture of the subclavian vein is about $1 \%$ in most studies. ${ }^{15-7} 910$ Consistent with previous studies, ${ }^{6-9}$ no pneumothoraces were observed when the cephalic vein was used for lead placement. Implantation of a DDD device significantly prolongs operation and fluoroscopy times. ${ }^{5} 10$ This difference is caused predominantly by the placement of the atrial lead, which is technically more challenging. However, among experienced operators the operation time for implantation of DDD pacemakers was only marginally longer than for single lead devices. Interestingly, implantation times of VVI and VDD devices were not significantly different, regardless of operator experience. Our VDD implantation strategy, which requires little additional time by testing different positions in the atrium, has been shown to minimise the incidence of atrial undersensing. ${ }^{15}$ Operation and fluoroscopy times were considerably shorter for operators with a high level of experience, particularly for DDD pacemaker systems. Operation and fluoroscopy times for operators with a low and medium level of experience were in the same range as those mentioned in the Danish pacemaker registry. ${ }^{5}$ In general, short implantation times are advantageous in terms of cost effectiveness and a putatively lower rate of infection. ${ }^{12}$

\section{Complication rates}

A wealth of data exists on early complication rates. ${ }^{1-11}$ However, recent data on late complications are sparse, ${ }^{56}$ particularly since complication rates must also be viewed in the light of progress in pacemaker technology. Improved implantation techniques, smaller and more durable leads, active fixation electrodes, and smaller pulse generators have been introduced, which makes it difficult to extrapolate historical data into the present. ${ }^{11}{ }^{19}$ We observed an early complication rate of $3.0 \%$ within the first three months after implantation and an overall complication rate of $4.5 \%$. This result is comparable with recent data from the Danish pacemaker registry, which also includes hospitals with small implantation volumes. ${ }^{5}$ The distribution of early complications is similar to that in other retrospective and prospective studies. ${ }^{1-11}$ Our data support the view that the cephalic cut down technique reduces early complications and incremental costs associated with the treatment of pneumothoraces after pacemaker implantation. ${ }^{9}$

RV dilatation has not been described as a predisposing factor for a higher complication rate. Complications arising from RV dilatation are (micro)dislocation of the ventricular lead, sensing failure, and exit block. Another factor predisposing to a higher complication rate was age $>74$ years. The impact of age on complication rates after pacemaker implantation has not been thoroughly investigated. Among patients enrolled in the PASE (pacemaker selection in the elderly) study, ${ }^{3}$ who were older than 65 years, $4.4 \%$ had complications requiring surgical revision, which is within the range of other prospective studies. In a smaller study, age $>80$ years did not predispose to a higher complication rate. ${ }^{20}$ The complication rate of older patients in our study was substantially higher with no predisposition to a specific complication. The reasons for this finding are speculative. Previous studies have reported a deterioration of atrial sensing with age $^{1621}$ and a higher incidence of unipolar ventricular pacemaker malfunction. ${ }^{17}$ This may be due to a certain degree of myocardial fibrosis in elderly patients. Moreover, we can hypothesise that elderly patients tend to be more agitated in the postoperative period and thus to be more prone to haematoma or early lead dislocation.

Late complications were observed in $1.5 \%$ of all patients at an annual complication rate of $0.5 \%$. The overall incidence of lead defects was low in our study ( $0.9 \%$ of all implantations), although we used a high proportion of bipolar leads in our study. In the Danish pacemaker registry, the eight year survival rate of bipolar ventricular leads was initially only $75 \%$, which was significantly lower than the survival rate for unipolar ventricular leads. ${ }^{22}$ However, with more recent bipolar electrode designs, the incidence of ventricular lead dysfunction for bipolar leads seems to be comparable with that for unipolar leads. ${ }^{23}$ The rate of lead dysfunction observed in our study is not higher than that in the Danish pacemaker registry, where a high proportion of unipolar ventricular leads was used. ${ }^{5}$ As an institutional standard, atrial active fixation leads were used in all cases. Complication rates of active and passive fixation leads have been 
systematically investigated in only a few small studies with no substantial differences. ${ }^{24} 25$ From a theoretical point of view, active fixation leads ought to have a lower dislocation rate. Although a higher incidence of exit block was described for active fixation leads, this does not apply to steroid eluting leads. ${ }^{26}$ There has been concern about the long term performance of the technically more complex VDD leads. ${ }^{13}{ }^{14}$ This is not supported by our data and other recent studies. ${ }^{21}{ }^{27}$ An infrequent but serious late complication is late perforation of a ventricular lead, an observation that was recently highlighted in a report by Ellenbogen and colleagues. ${ }^{28}$ We observed this late complication in a single patient during our study period.

\section{Influence of operator experience}

Concordant with previous reports, ${ }^{8-10}$ the complication rate decreased significantly with a higher level of operator experience. Interestingly, the influence of experience on the complication rate differed considerably according to the pacemaker system implanted. Complication rates were considerably lower for VVI and VDD systems than for DDD systems among operators with a low or medium level of experience but not with a high level of experience. The higher complication rates for dual chamber pacing in some prospective studies may thus be explained by a high proportion of inexperienced operators. ${ }^{17}$ This view is supported by two other studies, which found similar complication rates for single and dual chamber pacemakers among experienced operators. ${ }^{9}{ }^{10}$ In our setting, which included learning curves of six operators, more than 100 pacemaker implantations seem to have been necessary to achieve a low complication rate. Independent of the pacemaker system implanted, the relative risk of acquiring a complication decreases by $0.6 \%$ with each implantation performed. Initially, there seems to be a linear correlation between the number of operations and the complication rate. Although complication rate does not seem to decrease further after 200 implantations, owing to the limited number of operators it is difficult to give an exact cut off point where the complication rate does not decrease further with increased operator experience. Remarkably, the initial learning curve for single lead VDD pacemaker implantation is steeper than for DDD systems, and implantation does not result in a higher complication rate than with VVI pacing even at a low level of operator experience. Since single lead VDD pacing offers AV synchrony levels comparable with DDD pacing, ${ }^{12-14} 29$ VDD pacing may be a good alternative for centres with low implantation volumes when AV synchronous pacing in AV block is to be achieved.

\section{Study limitations}

The results presented in this study are based on the database of a German university hospital that serves as a referral centre. Institutional preferences, selection of specific pulse generators and leads, volume of implantations each year, and individual experience and skills may have influenced the results of our study. All operations in our study were performed in an operating theatre by cardiologists. Previous studies suggest that operations performed by surgeons in the operating theatre or by cardiologists in a catheterisation laboratory yield comparable complication rates. ${ }^{30-32}$ As the merits of VDD pacing were systematically investigated at our institution, a relatively high number of VDD devices were implanted during the study period..$^{29}$ Thus, complication rates of VDD pacing may be higher at institutions with less expertise for VDD pacing. The perioperative antibiotic regimen may also have an impact on postoperative infections. ${ }^{33}$ The effect of different antibiotic regimens on complication rates cannot be derived from our data, as the total number of infections was low and the antibiotic regimen was not consistent during the study period. Some patients may have been lost to follow up and might have sought treatment at other hospitals for their respective complication. Since we considered only complications that required an operative revision, less severe complications of pacemaker implantation were not registered.

\section{Conclusions}

Patient morbidity influences implantation times and complication rates. Particularly, RV dilatation, reduced LV function, and patient age predispose to higher complication rates. This study shows in a large patient cohort that operation and fluoroscopy times, as well as complication rates, depend on operator experience. Operators with a high level of experience do not seem to be associated with the higher complication rates for DDD pacemakers found in recent multicentre trials. If physiological pacing in AV block is to be achieved, VDD pacing offers an interesting alternative, since the learning curve for VDD systems among inexperienced operators seems to be similar to that for VVI systems.

\section{Authors' affiliations}

F Eberhardt, F Bode, H Bonnemeier, F Boguschewski, M Schlei, W Peters, U K H Wiegand, Universitätsklinik Schleswig Holstein, Campus Luebeck, Medizinische Klinik II, Luebeck, Germany

\section{REFERENCES}

1 Connolly SJ, Kerr CR, Gent M, et al. Effects of physiologic pacing versus ventricular pacing on the risk of stroke and death due to cardiovascular causes. Canadian trial of physiologic pacing investigators. N Engl J Med 2000;342:1385-91.

2 Andersen HR, Thuesen L, Bagger JP, et al. Prospective randomised trial of atrial versus ventricular pacing in sick sinus syndrome. Lancet 1994;344:1523-8.

3 Link MS, Estes NA 3rd, Griffin JJ, et al. Complications of dual chamber pacemaker implantation in the elderly. Pacemaker selection in the elderly (PASE) investigators. J Interv Card Electrophysiol 1998;2:175-9.

4 Lamas GA, Lee KL, Sweeney MO, et al. Ventricular pacing or dual-chamber pacing for sinus-node dysfunction. N Engl J Med 2002;346:1854-62.

5 Moller M, Arnsbo P, Asklund M, et al. Quality assessment of pacemaker implantation in Denmark. Europace 2002;4:107-12.

6 Kiviniemi MS, Pirnes MA, Eranen HJ, et al. Complications related to permanent pacemaker therapy. Pacing Clin Electrophysiol 1999;22:71 1-20.

7 Chauhan A, Grace AA, Newell SA, et al. Early complications after dual chamber versus single chamber pacemaker implantation. Pacing Clin Electrophysiol 1994;17:2012-5.

8 Parsonnet V, Bernstein AD, Lindsay B. Pacemaker-implantation complication rates: an analysis of some contributing factors. J Am Coll Cardiol 1989;13:917-21.

9 Tobin K, Stewart J, Westveer D, et al. Acute complications of permanent pacemaker implantation: their financial implication and relation to volume and operator experience. Am J Cardiol 2000;85:774-6.

10 Aggarwal RK, Connelly DT, Ray SG, et al. Early complications of permanent pacemaker implantation: no difference between dual and single chamber systems. Br Heart J 1995;73:571-5.

11 Moller JE, Simonsen EH, Moller M. Impact of continuous quality improvement of pacing mode and rate of complications in permanent pacing. Heart 1997;77:357-62.

12 Wiegand U, Potratz J, Bode F, et al. Cost-effectiveness of dual-chamber pacemaker therapy: does single lead VDD pacing reduce treatment costs of atrioventricular block? Eur Heart J 2001 ;22:174-80

13 Crick JC. European multicenter prospective follow-up study of 1,002 implants of a single lead VDD pacing system. The European multicenter study group. Pacing Clin Electrophysiol 1991;14:1742-4.

14 Curzio G. A multicenter evaluation of a single-pass lead VDD pacing system. The multicenter study group. Pacing Clin Electrophysio 1991; 14:434-42

15 Wiegand UK, Nowak B, Reisp U, et al. Implantation strategy of the atrial dipole impacts atrial sensing performance of single lead VDD pacemakers. Pacing Clin Electrophysiol 2002;25:316-23.

16 Sugiura T, Iwasaka T, Shiomi K, et al. Clinical significance of right ventricular dilatation with right ventricular infarction. Coron Artery Dis 1994;5:955-9.

17 Wiegand UK, Bode F, Bonnemeier $\mathrm{H}$, et al. Incidence and predictors of pacemaker dysfunction with unipolar ventricular lead configuration. Can we identify patients who benefit from bipolar electrodes? Pacing Clin Electrophysiol 2001;24:1383-8.

18 Brandt J, Attewell R, Fahraeus T, et al. Acute atrial endocardial P wave amplitude and chronic pacemaker sensitivity requirements: relation to patient age and presence of sinus node disease. Pacing Clin Electrophysiol 1990;13:417-24. 
19 Karnatz P, Elsner C, Mueller G, et al. Permanent pacemaker therapy before and after the reunification of Germany: 16 years of experience at an East German regional pacing center. Pacing Clin Electrophysiol 2000;23:991-7.

20 Rosenheck S, Geist $M$, Weiss A, et al. Permanent cardiac pacing in octogenarians. Am J Geriatr Cardiol 1995;4:42-7.

21 Wiegand UK, Potratz J, Bode F, et al. Age dependency of sensing performance and AV synchrony in single lead VDD pacing. Pacing Clin Electrophysiol 2000;23:863-9.

22 Moller M, Arnsbo P. Appraisal of pacing lead performance from the Danish pacemaker register. Pacing Clin Electrophysiol 1996;19:1327-36.

23 Arnsbo P, Moller M. Updated appraisal of pacing lead performance from the Danish pacemaker register: the reliability of bipolar pacing leads has improved. Pacing Clin Electrophysiol 2000;23:1401-6.

24 Connelly DT, Steinhaus DM, Handlin L, et al. Atrial pacing leads following open heart surgery: active or passive fixation? Pacing Clin Electrophysiol 1997;20:2429-33.

25 Schuchert A, van Langen $\mathrm{H}$, Michels $\mathrm{K}$, et al. [Comparison of active and passive fixation of steroid emitting atrial electrodes]. Z Kardiol 1996:85:255-59 (In German).

26 Wiegand UK, Potratz J, Bonnemeier $\mathrm{H}$, et al. Electrophysiological performance of a bipolar membrane-coated titanium nitride electrode: a randomized comparison of steroid and nonsteroid lead designs. Pacing Clin Electrophysiol 1999;22:935-41.
27 Chamberlain-Webber $\mathbf{R}$, Barnes $E$, Papouchado $M$, et al. Long-term survival of VDD pacing. Pacing Clin Electrophysiol 1998;21:2246-8.

28 Ellenbogen KA, Wood MA, Shepard RK. Delayed complications following pacemaker implantation. Pacing Clin Electrophysiol 2002;25:1155-8.

29 Wiegand UK, Bode F, Schneider R, et al. Atrial sensing and AV synchrony in single lead VDD pacemakers: a prospective comparison to DDD devices with bipolar atrial leads. J Cardiovasc Electrophysiol 1999;10:513-20.

30 Yamamura KH, Kloosterman EM, Alba J, et al. Analysis of charges and complications of permanent pacemaker implantation in the cardiac catheterization laboratory versus the operating room. Pacing Clin Electrophysiol 1999;22:1820-4.

31 Miller GB, Leman RB, Kratz JM, et al. Comparison of lead dislodgment and pocket infection rates after pacemaker implantation in the operating room versus the catheterization laboratory. Am Heart $J$ 1988; 115:1048-51

32 Stamato NJ, O'Toole MF, Enger EL. Permanent pacemaker implantation in the cardiac catheterization laboratory versus the operating room: an analysis of hospital charges and complications. Pacing Clin Electrophysiol 1992;15:2236-9.

33 Mounsey JP, Griffith MJ, Tynan M, et al. Antibiotic prophylaxis in permanent pacemaker implantation: a prospective randomised trial. Br Heart $J$ 1994:72:339-43.

\section{IMAGES IN CARDIOLOGY}

\section{Secondary collapse of a Palmaz stent implanted for native aortic coarctation}

A

21 year old woman was referred for native aortic coarctation. At 6 months of age she had had a repair of an aortopulmonary window, at 12 months a surgical closure of an atrial septal defect, and at 7 years a left pneumonectomy. During the first years of life aortic coarctation was not discovered.

At presentation she had systemic hypertension and superior-inferior limb gradient of $30 \mathrm{~mm} \mathrm{Hg}$. Echocardiography and magnetic resonance imaging confirmed aortic coarctation. Direct stenting of the coarcted segment was performed utilising a Palmaz 308 stent mounted on an $18 \mathrm{~mm}$ balloon. After stenting there was no residual gradient and the profile of the aortic hysthmus was regular.

Six months later the patient still had systemic hypertension and a superior-inferior limb gradient of $40 \mathrm{~mm} \mathrm{Hg}$. Computed tomography showed deviation of the heart, deformation (panel A), and possible rupture (panel B) of the stent, and did not identify any cause of extrinsic compression.

The risk of surgical repair of coarctation was considered to be elevated, thus, redilation of the stent was attempted, utilising a $18 \mathrm{~mm}$ balloon. Peak to peak gradient diminished from 50 to $20 \mathrm{~mm} \mathrm{Hg}$ and aortic profile appeared more regular.

Two months after the second catheterisation systemic hypertension persisted and there was a superior-inferior limb gradient of $50 \mathrm{~mm} \mathrm{Hg}$. Computed tomography showed an incompletely expanded stent, with a transversal diameter of $8.8 \mathrm{~mm}$ (panel C) and an axial diameter of $4.6 \mathrm{~mm}$ (panel D).

In June 2003, the patient had implantation of a right conduit between the ascending and descending aorta. At last follow up she was asymptomatic and had a normal aortic pressure.
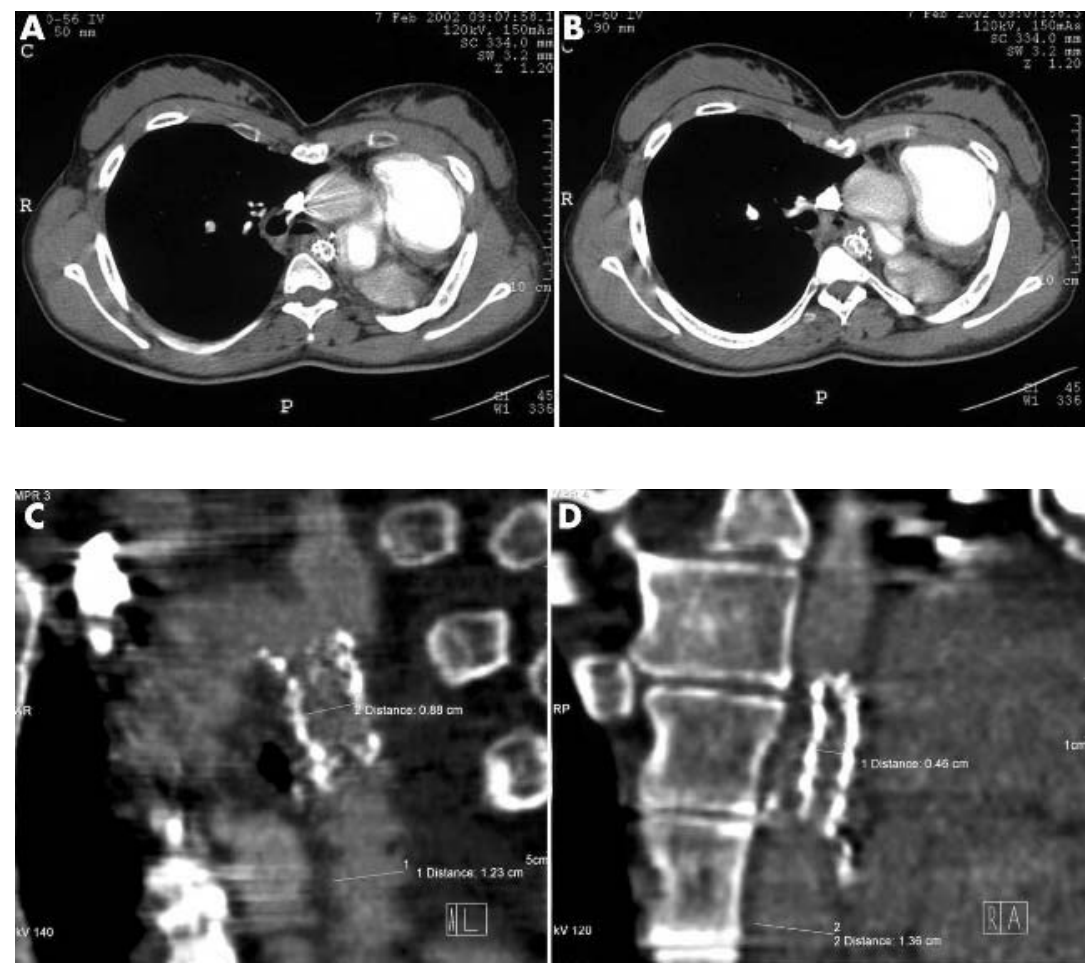

We are unable to explain the reasons for the repeated stent collapse, apart from a possible compression between the spine and the beating heart. 